\title{
LIOUVILLE TYPE THEOREM FOR NONLINEAR BOUNDARY VALUE PROBLEM ON HEISENBERG GROUP
}

\author{
Xiaojun Zhao \\ Peking University, School of Economics \\ Beijing, 100871, P. R. China; zhaoxiaojun@pku.edu.cn
}

\begin{abstract}
In this paper, we establish some Liouville type theorem for nonlinear elliptic equation in the Heisenberg group with nonlinear boundary value condition. We use the moving plane method to prove our result.
\end{abstract}

\section{Introduction}

Liouville type theorems are very important in proving the priori bound for elliptic equations. More precisely, to prove the priori bound, we usually use the blow up method. After blowing up, we get a bounded solution for the limit equation, this contradicts the nonexistence result of the limit equation. Hence, the priori bound follows.

In the past few years, Liouville theorems for nonlinear elliptic equation on Heinsenberg have attracted much attention of mathematicians. For example, in the paper [1], the authors considered the following elliptic inequality on the Heisenberg group $H^{n}$

$$
\Delta_{H} u+u^{p} \leq 0 \quad \text { in } H^{n} .
$$

They proved that problem (1.1) possesses only trivial non-negative solution for $1<$ $p \leq \frac{Q}{Q-2}$, where $Q=2 n+2$ is the homogeneous dimension of $H^{n}$, a similar result for the Laplacian equations on $\mathbf{R}^{N}$. Moreover, the authors also proved the exponent $\frac{Q}{Q-2}$ is optimal for this inequality in the sense that, if $p>\frac{Q}{Q-2}$, then problem (1.1) indeed has a nontrivial positive solution. After the work of [1], there are plenty of works concerning on the nonexistence results for the following elliptic equation rather than elliptic inequality

$$
\Delta_{H} u+u^{p}=0 \text { in } H^{n} .
$$

In the Euclidean case, nonexistence of positive solution for equation

$$
\Delta u+u^{p}=0 \quad \text { in } \mathbf{R}^{N}
$$

has been established by Gidas and Spruck in [10]. They proved that problem (1.3) does not possess positive solutions for $0<p<\frac{N+2}{N-2}$. The proof of this result was simplified by Chen and Li by using the moving plane method in [5]. Moreover, in both papers, they proved that the exponent $\frac{N+2}{N-2}$ is optimal. Inspired by results for the Euclidean case, a natural question is that whether similar nonexistence result holds for problem (1.2) with $0<p<\frac{Q+2}{Q-2}$. However, this problem has not been completely solved yet, we only mention some partial results here. After the work [1], Birindelli

https://doi.org/10.5186/aasfm.2017.4243

2010 Mathematics Subject Classification: Primary 35J60, 35R03, 35J15.

Key words: Liouville type theorem, moving plane method, Heisenberg group. 
and Prajapat studied the nonexistence result for cylindrical solution in [3], they proved problem (1.2) does not possess positive cylindrical solutions providing $0<p<$ $\frac{Q+2}{Q-2}$. Here we say that $u$ is cylindrical in $H^{n}$ if for any $(x, y, t) \in H^{n}$, where $(x, y) \in$ $\mathbf{R}^{N} \times \mathbf{R}^{N}$ and $t \in \mathbf{R}$ is the anisotropic direction, we have $u(x, y, t)=u(r, t)$ with $r=\sqrt{x^{2}+y^{2}}$. They used the moving plane method to prove this result. Although the Heisenberg Laplacian satisfies the maximum principle, it is not invariant by the usual reflection with respect to a hyperplane. In order to apply the moving plane method, the authors introduced a new reflection called the "H-reflection" for the Heisenberg Laplacian. Because the Heisenberg Laplacian is invariant with respect to the H-reflection only for cylindrical solution, so the authors only obtained the Liouville theorem for cylindrical solutions. After this work, in order to solve the nonexistence result for any positive solution, much attentions have been paid to prove that any positive solution for problem (1.2) is cylindrical symmetric. But up to now, this conjecture has not been solved completely yet, partial results on this aspect can be found in $[2,4,9]$ and etc.

Besides using the moving plane method, other methods were also used to prove the nonexistence results. For example, $\mathrm{Xu}$ [17] used the vector field method to study the nonexistence result for the following equation

$$
\Delta_{H} u+h(x) u^{p}=0 \text { in } H^{n} .
$$

Under some assumptions on the weight $h(x)$, she proved equation (1.4) possesses no positive solutions providing $1<p<1+\frac{8 n+7}{(2 n+1)^{2}}$. We note the exponent $1+\frac{8 n+7}{(2 n+1)^{2}}$ is smaller than $\frac{Q+2}{Q-2}$.

Recently, in order to overcome the nonlocal property of the fractional Heisenberg Laplacian equations

$$
\mathcal{P}_{\frac{1}{2}} u=u^{p} \quad \text { in } H^{n},
$$

Frank, González, Monticelli and Tan [8] studied the extended problem on $H^{n} \times \mathbf{R}^{+}$. More precisely, they proved problem (1.5) is equivalent to the following local problem

$$
\begin{cases}\frac{\partial^{2} U}{\partial \lambda^{2}}+\lambda^{2} \frac{\partial U^{2}}{\partial t^{2}}+\frac{1}{2} \Delta_{H} U=0 & \text { in } \hat{H}_{+}^{n}=H^{n} \times \mathbf{R}^{+}, \\ -c \frac{\partial U}{\partial \lambda}=U(., 0)^{p} & \text { on } \partial \hat{H}_{+}^{n}=H^{n} \times\{0\},\end{cases}
$$

where $c$ is a positive constant depending only on $n$. In a sequencing paper [6], Cinti and Tan proved the nonexistence of positive solution for problem equation (1.6). More precisely, they proved problem (1.6) possesses no positive cylindrical solutions provided $0<p<\frac{Q+1}{Q-1}$, where $Q=2 n+2$ is the homogenous dimension of $H^{n}$.

In this paper, we study another problem

$$
\begin{cases}\frac{\partial^{2} u}{\partial \lambda^{2}}+4 \lambda^{2} \frac{\partial u^{2}}{\partial t^{2}}+\Delta_{H} u+f(u)=0 & \text { in } \hat{H}_{+}^{n}=H^{n} \times \mathbf{R}^{+}, \\ -\frac{\partial u}{\partial \lambda}=g(u) & \text { on } \partial \hat{H}_{+}^{n}=H^{n} \times\{0\}\end{cases}
$$

i.e., both the equation and the boundary condition are nonlinear. Our main result is the following

Theorem 1.1. Suppose that $u \in C^{0}\left(\hat{H}_{+}^{n}\right)$ is a nonnegative cylindrical solution for problem (1.7), i.e., $u(x, y, t, \lambda)=u\left(r_{0}, t, \lambda\right)$ with $r_{0}=\sqrt{x^{2}+y^{2}}, f, g:[0,+\infty) \rightarrow$ $[0,+\infty)$ are continuous functions with the following properties:

(i) $f(t), g(t)$ are nondecreasing in $(0,+\infty)$,

(ii) $h(t)=\frac{f(t)}{t^{\frac{Q}{Q}-1}}, k(t)=\frac{g(t)}{t^{\frac{Q+1}{Q-1}}}$ are nonincreasing in $(0,+\infty)$, 
(iii) either $h$ or $k$ is not a constant.

Then $u \equiv c$ with $f(c)=g(c)=0$.

We want to use the moving plane method to prove our result. However, since the nonlinearities are only assumed to be continuous rather than Lipschitz continuous, the solutions are generally not of $C^{2}$ class. As a result, the usual maximum principle in a differential form does not work. In order to overcome this difficulty, we use some integral inequality to substitute the maximum principle, which was first introduced by Terracini in $[15,16]$. After Terracini's work, moving plane method based on integral inequalities was widely used to prove Liouville theorems. For example, Damascelli and Gladiali [7] proved some nonexistence results for the Laplacian equations with general nonlinearities. Guo and Liu studied the general Liouville type theorem for elliptic system and biharmonic equation in $[12,13,11]$ respectively. Yu studied the nonlinear Liouville type theorem for integral equation and integral system in [18]. Other results can be found in $[19,20]$ and etc.

The rest of this paper is devoted to the proof of Theorem 1.1. For completeness, we first give some preliminaries on Heisenberg group in Section 2. The proof of Theorem 1.1 is finally finished in Section 3. In the following, we denote by $C$ some constant, which may vary from line to line.

\section{Preliminaries}

In this section, we collect some basic properties concerning the Heisenberg group $H^{n}$ and its extension $\hat{H}_{+}^{n}$. We also give some results on the operators $\Delta_{H}$ and $L:=\frac{\partial^{2}}{\partial \lambda^{2}}+4 \lambda^{2} \frac{\partial^{2}}{\partial t^{2}}+\Delta_{H}$. For proofs and more information we refer for example to $[12,14,8]$.

For elements in $H^{n}$, we shall use the notation $\xi=(x, y, t) \in \mathbf{R}^{N} \times \mathbf{R}^{N} \times \mathbf{R}$, where $x=\left(x_{1}, x_{2}, \ldots, x_{n}\right), y=\left(y_{1}, y_{2}, \ldots, y_{n}\right)$. The Heisenberg group $H^{n}$ is the space $\mathbf{R}^{2 n+1}$ endowed with the group action $\circ$ defined by

$$
\xi_{0} \circ \xi=\left(x+x_{0}, y+y_{0}, t+t_{0}+2 \sum_{i=1}^{n}\left(x_{i} y_{i_{0}}-y_{i} x_{i_{0}}\right)\right),
$$

where $\xi=(x, y, t)$ and $\xi_{0}=\left(x_{0}, y_{0}, t_{0}\right)$. Let us denote by $\delta_{\lambda}$ the parabolic dilation in $\mathbf{R}^{2 n+1}$, i.e.,

$$
\delta_{\lambda}(\xi)=\left(\lambda x, \lambda y, \lambda^{2} t\right),
$$

then it is easy to see $\delta_{\lambda}\left(\xi_{0} \circ \xi\right)=\delta_{\lambda}\left(\xi_{0}\right) \circ \delta_{\lambda}(\xi)$. Moreover, the norm of $H^{n}$ defined by

$$
\rho=\|\xi\|=\left[\left(\sum_{i=1}^{n}\left(x_{i}^{2}+y_{i}^{2}\right)\right)^{2}+t^{2}\right]^{\frac{1}{4}}
$$

is homogeneous of degree one with respect to the dilation $\delta_{\lambda}$.

The associated distance between two points $\xi, \xi_{0}$ of $H^{n}$ is defined accordingly by

$$
d\left(\xi, \xi_{0}\right)=\left\|\xi_{0}^{-1} \circ \xi\right\|,
$$

where $\xi_{0}^{-1}$ is the inverse of $\xi_{0}$ with respect to $\circ$. The open ball of radius $R$ centered at $\xi_{0}$ is defined by

$$
B\left(\xi_{0}, R\right)=\left\{\xi \in H^{n}: d\left(\xi, \xi_{0}\right)<R\right\} .
$$

A direct calculation shows that

$$
\left|B\left(\xi_{0}, R\right)\right|=|B(0, R)|=R^{Q}|B(0,1)|,
$$


where $Q=2 n+2$ and $|\cdot|$ denotes the Lebesgue measure. The number $Q$ is usually called the homogeneous dimension of $H^{n}$.

To define the gradient and the Heisenberg Laplacian of a function in $H^{n}$, we introduce the following vector fields

$$
\begin{aligned}
X_{i} & =\frac{\partial}{\partial x_{i}}+2 y_{i} \frac{\partial}{\partial t}, \quad i=1,2, \ldots, n \\
Y_{i} & =\frac{\partial}{\partial y_{i}}-2 x_{i} \frac{\partial}{\partial t}, \quad i=1,2, \ldots n
\end{aligned}
$$

and

$$
T=\frac{\partial}{\partial t}
$$

Then $\left\{X_{i}, Y_{i}, T\right\}(i=1,2, \ldots, n)$ form a base of the Lie algebra of vector fields which is left invariant with respect to the Heisenberg action $\circ$. The Heisenberg gradient of a function $u$ is defined by

$$
\nabla_{H} u=\left(X_{1} u, X_{2} u, \ldots, X_{n} u, Y_{1} u, Y_{2} u, \ldots, Y_{n} u\right) .
$$

Moreover, the Heisenberg Laplacian $\Delta_{H}$ is defined by

$$
\Delta_{H}=\sum_{i=1}^{n}\left(X_{i}^{2}+Y_{i}^{2}\right)
$$

A direct calculations show that

$$
\Delta_{H}=\sum_{i=1}^{n}\left(\frac{\partial^{2}}{\partial x_{i}^{2}}+\frac{\partial^{2}}{\partial y_{i}^{2}}+4 y_{i} \frac{\partial^{2}}{\partial x_{i} \partial t}-4 x_{i} \frac{\partial^{2}}{\partial y_{i} \partial t}+4\left(x_{i}^{2}+y_{i}^{2}\right) \frac{\partial^{2}}{\partial t^{2}}\right) .
$$

For cylindrical function $u(r, t)$, the above formula can be simplified to

$$
\Delta_{H} u(r, t)=\frac{\partial^{2} u}{\partial r^{2}}+\frac{2 n-1}{r} \frac{\partial u}{\partial r}+4 r^{2} \frac{\partial^{2} u}{\partial t^{2}} .
$$

Analogously to $H^{n}$, in $\hat{H}_{+}^{n}$, we define the following group law: for $z=\left(x_{1}, \ldots, x_{n}\right.$, $\left.y_{1}, \ldots, y_{n}, t, \lambda\right) \in \hat{H}_{+}^{n}$ and $\hat{z}=\left(\hat{x}_{1}, \ldots, \hat{x}_{n}, \hat{y}_{1}, \ldots, \hat{y}_{n}, \hat{t}, \hat{\lambda}\right) \in \hat{H}_{+}^{n}$, we define

$$
\hat{z} \circ z:=\left(\hat{x}+x, \hat{y}+y, \hat{t}+t+2 \sum_{j=1}^{n}\left(x_{j} \hat{y}_{j}-y_{j} \hat{x}_{j}\right), \hat{\lambda}+\lambda\right) .
$$

Moreover, the norm of $z \in \hat{H}_{+}^{n}$ is given by

$$
\|z\|=\left[\left(|x|^{2}+|y|^{2}+\lambda^{2}\right)^{2}+t^{2}\right]^{\frac{1}{4}}
$$

and the distance $d_{\hat{H}}$ between $z$ and $\hat{z}$ is defined by

$$
d_{\hat{H}}(z, \hat{z}):=\left\|\hat{z}^{-1} \circ z\right\| \text {. }
$$

Observe that when $\lambda=\hat{\lambda}=0$, that is, $z$ and $\hat{z}$ belong to $H^{n}$, then $d_{\hat{H}}(z, \hat{z})=d_{H}(z, \hat{z})$. Similarly, for any given $\hat{z} \in \hat{H}_{+}^{n}$, we define the open ball with center at $\hat{z}$ and radius $R$ as

$$
B(\hat{z}, R)=\left\{z \in \hat{H}_{+}^{n} \mid d_{\hat{H}}(z, \hat{z})<R\right\}
$$

and for $z_{0} \in H^{n} \times\{0\}$, we denote

$$
B^{+}\left(z_{0}, R\right)=\left\{z \in \hat{H}_{+}^{n} \mid d_{\hat{H}}\left(z, z_{0}\right)<R, \lambda>0\right\} .
$$


Hence, with the above notations, the operator $L$ can be written explicitly as

$$
L=\frac{\partial^{2}}{\partial \lambda^{2}}+\sum_{j=1}^{n}\left(\frac{\partial^{2}}{\partial x_{j}^{2}}+\frac{\partial^{2}}{\partial y_{j}^{2}}+4 y_{j} \frac{\partial^{2}}{\partial x_{j} \partial t}-4 x_{j} \frac{\partial^{2}}{\partial y_{j} \partial t}\right)+4\left(\lambda^{2}+\sum_{j=1}^{n}\left(x_{j}^{2}+y_{j}^{2}\right)\right) \frac{\partial^{2}}{\partial t^{2}} .
$$

The operator $L$ can also be written as $L=\operatorname{div}\left(A \nabla^{T}\right)$, where $A$ is a $(2 n+2) \times(2 n+2)$ symmetric matrix with $a_{k j}=\delta_{k j}$ for $k, j=1,2, \ldots, 2 n, a_{j, 2 n+1}=a_{2 n+1, j}=2 y_{j}$ for $j=1,2, \ldots, n, a_{j, 2 n+1}=a_{2 n+1, j}=-2 x_{j}$ for $j=n+1, n+2, \ldots, 2 n, a_{2 n+1,2 n+1}=$ $4\left(|x|^{2}+|y|^{2}+\lambda^{2}\right), a_{2 n+2,2 n+2}=1$ and $a_{j, 2 n+2}=a_{2 n+2, j}=0$ for $j=1,2, \ldots, 2 n+1$.

We finally introduce the CR inversion on $\hat{H}_{+}^{n}$ to end this section, which will be used later. As in [6], for any $(x, y, t, \lambda) \in \hat{H}_{+}^{n}$, we denote $r=\left(|x|^{2}+|y|^{2}+\lambda^{2}\right)^{\frac{1}{2}}$ and $\rho=\left(r^{4}+t^{2}\right)^{\frac{1}{4}}$. Also, we set

$$
\tilde{x}_{i}=\frac{x_{i} t+y_{i} r^{2}}{\rho^{4}}, \quad \tilde{y}_{i}=\frac{y_{i} t-x_{i} r^{2}}{\rho^{4}}, \quad \tilde{t}=-\frac{t}{\rho^{4}}, \quad \tilde{\lambda}=\frac{\lambda}{\rho^{2}} .
$$

We define the CR inversion of a regular function $u(x, y, t, \lambda)$ in $\hat{H}_{+}^{n}$ as

$$
v(x, y, t, \lambda)=\frac{1}{\rho^{Q-1}} u(\tilde{x}, \tilde{y}, \tilde{t}, \tilde{\lambda}) .
$$

As for $u$ and its CR inversion $v$, we have the following

Lemma 2.1. Suppose that $u \in C^{2}\left(\hat{H}_{+}^{n}\right) \cap C\left(\overline{\hat{H}_{+}^{n}}\right)$ is a solution of $(1.7)$, then the $C R$ inversion $v$ of $u$ satisfies

$$
\begin{cases}-L v=\frac{f(u)}{\rho^{Q+3}} & \text { in } \quad \hat{H}_{+}^{n} \backslash\{0\} \\ -\frac{\partial v}{\partial \lambda}=\frac{g(u)}{\rho^{Q+1}} & \text { on } \partial \hat{H}_{+}^{n} \backslash\{0\} .\end{cases}
$$

For the proof of this Lemma, please see the proof of Lemma 3.1 in [6].

\section{Proof of Theorem 1.1}

With the above notations and preliminaries, we can prove Theorem 1.1 now. We want to use the moving plane method to prove our result. The first step of moving plane is to show that this procedure can be started at some point. Since we don't know the decay behaviors of $u$, it seems impossible to use this method directly on $u$. As one usually use the Kelvin transformation in Euclidean case, we turn to the CR inversion function $v$ of $u$.

We recall that $v$, the $\mathrm{CR}$ inverse of $u$ at $(0,0,0,0)$, is defined by

$$
v(x, y, t, \lambda)=\frac{1}{\rho^{Q-1}} u(\tilde{x}, \tilde{y}, \tilde{t}, \tilde{\lambda}) .
$$

From the definition, we know that $v$ is continuous in $\hat{H}_{+}^{n} \backslash\{0\}$ and $v$ decays to zero at infinity. Moreover, we have the following decay estimate

$$
\lim _{\rho \rightarrow \infty} \rho^{Q-1} v(r, t, \lambda)=u(0) .
$$

Since $u$ is a cylindrical function and satisfies the equation (1.7), then $v$ is also cylindrical and satisfies

$$
\begin{cases}-L v=\frac{f(u)}{\rho^{Q+3}} & \text { in } \quad \hat{H}_{+}^{n} \backslash\{0\} \\ -\frac{\partial v}{\partial \lambda}=\frac{g(u)}{\rho^{Q+1}} & \text { on } \quad \partial \hat{H}_{+}^{n} \backslash\{0\}\end{cases}
$$


by Lemma 2.1. If we define $h(t)=\frac{f(t)}{t^{\frac{Q}{Q-1}}}$ and $k(t)=\frac{g(t)}{t^{\frac{Q}{Q}-1}}$, then the above equation turns to be

$$
\begin{cases}-L v=h\left(\rho^{Q-1} v\right) v^{\frac{Q+3}{Q-1}} & \text { in } \quad \hat{H}_{+}^{n} \backslash\{0\}, \\ -\frac{\partial v}{\partial \lambda}=k\left(\rho^{Q-1} v\right) v^{\frac{Q+1}{Q-1}} & \text { on } \partial \hat{H}_{+}^{n} \backslash\{0\} .\end{cases}
$$

Now we can start the moving plane method on the function $v$. First, we give the environment we will work in. Let $\Sigma_{\mu}=\left\{(x, y, t, \lambda) \in \hat{H}_{+}^{n} \mid t \geq \mu\right\}, T_{\mu}=\{(x, y, t, \lambda) \in$ $\left.\hat{H}_{+}^{n} \mid t=\mu\right\}$ and $p_{\mu}=(0,0,2 \mu, 0)$. As in [6], we define the H-reflection function $v_{\mu}$ of $v$ with respect to $T_{\mu}$ on $\Sigma_{\mu}$ by

$$
v_{\mu}(x, y, t, \lambda)=v_{\mu}\left(r_{0}, t, \lambda\right)=v\left(r_{0}, 2 \mu-t, \lambda\right)=v(y, x, 2 \mu-t, \lambda)
$$

for any $(x, y)$ such that $\left(x^{2}+y^{2}\right)^{\frac{1}{2}}=r_{0}$. Then we can infer from equation (3.3) that $v_{\mu}$ satisfies

$$
\left\{\begin{array}{l}
-L v_{\mu}=h\left(\rho_{\mu}^{Q-1} v_{\mu}\right) v_{\mu}^{\frac{Q+3}{Q-1}} \quad \text { in } \hat{H}_{+}^{n} \backslash\left\{p_{\mu}\right\}, \\
-\frac{\partial v_{\mu}}{\partial \lambda}=k\left(\rho_{\mu}^{Q-1} v_{\mu}\right) v_{\mu}^{\frac{Q+1}{Q-1}} \quad \text { on } \partial \hat{H}_{+}^{n} \backslash\left\{p_{\mu}\right\} .
\end{array}\right.
$$

If we defined $w_{\mu}=v-v_{\mu}$, then we have the following key lemma.

Lemma 3.1. For any fixed $\mu>0$, we have $w_{\mu}^{+}, v \in L^{2^{\#}}\left(\Sigma_{\mu}\right) \cap L^{\infty}\left(\Sigma_{\mu}\right)$ with $2^{\#}=\frac{2(Q+1)}{Q-1}$. Further more, there exists $C_{\mu}>0$, which is nonincreasing in $\mu$, such that

$$
\begin{aligned}
& \int_{\Sigma_{\mu}}\left|\nabla_{\hat{H}}\left(v-v_{\mu}\right)^{+}\right|^{2} d z \\
& \leq C_{\mu}\left[\left(\int_{A_{\mu}} \frac{1}{|\rho|^{2 Q+2}}\right)^{\frac{2}{Q+1}}+\left(\int_{B_{\mu}} \frac{1}{|\rho|^{2 Q}}\right)^{\frac{1}{Q}}\right] \cdot\left(\int_{\Sigma_{\mu}}\left|\nabla_{\hat{H}}\left(v-v_{\lambda}\right)^{+}\right|^{2} d z\right)
\end{aligned}
$$

where $\nabla_{\hat{H}}=\left(\frac{\partial}{\partial \lambda}, 2 \lambda \frac{\partial}{\partial t}, X_{1}, X_{2}, \ldots, X_{n}, Y_{1}, Y_{2}, \ldots, Y_{n}\right)$ and $A_{\mu}=\left\{(x, y, t, \lambda) \in \Sigma_{\mu} \mid v>\right.$ $\left.v_{\mu}\right\}, B_{\mu}=\left\{(x, y, t, \lambda) \in \partial \Sigma_{\mu} \mid v>v_{\mu}, \lambda=0\right\}$.

Proof. Since $\mu>0$, there exists $r>0$ such that $\Sigma_{\mu} \subset \hat{H}_{+}^{n} \backslash B_{r}(0)$. By the decay estimate of $v$, see equation (3.1), we have $v$ and hence $\left(v-v_{\mu}\right)^{+} \leq v \in$ $L^{2^{\#}}\left(\Sigma_{\mu}\right) \cap L^{\infty}\left(\Sigma_{\mu}\right)$. Now, we choose a cylinder symmetric cut-off function $0 \leq \eta_{\varepsilon} \leq 1$ such that

$$
\eta_{\varepsilon}= \begin{cases}1 & \text { if } \quad 2 \varepsilon \leq\left\|z \circ p_{\mu}^{-1}\right\| \leq \frac{1}{\varepsilon} \\ 0 & \text { if } \quad\left\|z \circ p_{\mu}^{-1}\right\| \leq \varepsilon \text { or }\left\|z \circ p_{\mu}^{-1}\right\| \geq \frac{2}{\varepsilon}\end{cases}
$$

$\left|\nabla_{\hat{H}} \eta\right| \leq \frac{C}{\varepsilon}$ for $\varepsilon<\left\|z \circ p_{\mu}^{-1}\right\| \leq 2 \varepsilon$ and $\left|\nabla_{\hat{H}} \eta\right| \leq C \varepsilon$ for $\frac{1}{\varepsilon}<\left\|z \circ p_{\mu}^{-1}\right\| \leq \frac{2}{\varepsilon}$.

Since $v$ and $v_{\mu}$ satisfy the following equations

$$
-L v(x, y, t)=h\left(\rho^{Q-1} v(x, y, t, \lambda)\right) v(x, y, t, \lambda)^{\frac{Q+3}{Q-1}}
$$

and

$$
-L v_{\mu}(x, y, t)=h\left(\rho_{\mu}^{Q-1} v_{\mu}(x, y, t, \lambda)\right) v_{\mu}(x, y, t, \lambda)^{\frac{Q+3}{Q-1}}
$$


we use $\psi=\eta_{\varepsilon}\left(v-v_{\mu}\right)^{+}$as a test function and denote $\phi=\eta_{\varepsilon}^{2}\left(v-v_{\mu}\right)^{+}$, then we get

$$
\begin{aligned}
& \int_{\Sigma_{\mu} \cap\left\{2 \varepsilon \leq\left\|z \circ p_{\mu}^{-1}\right\| \leq \frac{1}{\varepsilon}\right\}}\left|\nabla_{\hat{H}}\left(v-v_{\mu}\right)^{+}\right|^{2} \leq \int_{\Sigma_{\mu}}\left|\nabla_{\hat{H}} \psi\right|^{2} \\
& =\int_{\Sigma_{\mu}} \nabla_{\hat{H}}\left(v-v_{\mu}\right)^{+} \nabla_{\hat{H}} \phi d z+\int_{\Sigma_{\mu}}\left[\left(v-v_{\mu}\right)^{+}\right]^{2}\left|\nabla_{\hat{H}} \eta_{\varepsilon}\right|^{2} d z \\
& =\int_{A_{\mu}}-L\left(v-v_{\mu}\right) \phi d z+\int_{B_{\mu}} \frac{\partial\left(v-v_{\mu}\right)}{\partial \nu} \phi d z^{\prime}+I_{\varepsilon} \\
& =\int_{A_{\mu}}\left[h\left(\rho^{Q-1} v\right) v^{\frac{Q+3}{Q-1}}-h\left(\rho_{\mu}^{Q-1} v_{\mu}\right) v_{\mu}^{\frac{Q+3}{Q-1}}\right] \phi d z \\
& \quad+\int_{B_{\mu}}\left[k\left(\rho^{Q-1} v\right) v^{\frac{Q+1}{Q-1}}-k\left(\rho_{\mu}^{Q-1} v_{\mu}\right) v_{\mu}^{\frac{Q+1}{Q-1}}\right] d z^{\prime}+I_{\varepsilon},
\end{aligned}
$$

where $I_{\varepsilon}=\int_{\Sigma_{\mu}}\left[\left(v-v_{\mu}\right)^{+}\right]^{2}\left|\nabla_{\hat{H}} \eta_{\varepsilon}\right|^{2} d z$. Since $h, k$ are nonincreasing functions, $\rho>\rho_{\mu}$ and $v(z)>v_{\mu}(z)$ in $A_{\mu}$ and $B_{\mu}$, we conclude that

$$
h\left(\rho^{Q-1} v\right) \leq h\left(\rho_{\mu}^{Q-1} v_{\mu}\right) \quad \text { and } \quad k\left(\rho^{Q-1} v\right) \leq k\left(\rho_{\mu}^{Q-1} v_{\mu}\right)
$$

in $A_{\mu}$ and $B_{\mu}$ respectively. Hence we have

$$
\begin{aligned}
& \int_{\Sigma_{\mu} \cap\left\{2 \varepsilon \leq\left\|z \circ p_{\mu}^{-1}\right\| \leq \frac{1}{\varepsilon}\right\}}\left|\nabla_{\hat{H}}\left(v-v_{\mu}\right)^{+}\right|^{2} \\
& \leq \int_{A_{\mu}} h\left(\rho^{Q-1} v\right)\left[v^{\frac{Q+3}{Q-1}}-v_{\mu}^{\frac{Q+3}{Q-1}}\right] \phi d z+\int_{B_{\mu}} k\left(\rho^{Q-1} v\right)\left[v^{\frac{Q+1}{Q-1}}-v_{\mu}^{\frac{Q+1}{Q-1}}\right] d z^{\prime}+I_{\varepsilon} .
\end{aligned}
$$

By the decay estimate of $v$, see equation (3.1), we conclude that the constant $C_{\mu}^{1}:=$ $\frac{Q+3}{Q-1} \sup \left\{h\left(\rho^{Q-1} v(x, y, t, \lambda)\right)+k\left(\rho^{Q-1} v(x, y, t, \lambda)\right) \mid t \geq \mu\right\}$ is well-defined for $\mu>0$ and is nonincreasing in $\mu$. So we deduce from the above inequality that

$$
\begin{aligned}
& \int_{\Sigma_{\mu} \cap\left\{2 \varepsilon \leq\left\|z \circ p_{\mu}^{-1}\right\| \leq \frac{1}{\varepsilon}\right\}}\left|\nabla_{\hat{H}}\left(v-v_{\mu}\right)^{+}\right|^{2} d z \\
& \leq C_{\mu}^{1}\left[\int_{A_{\mu}} v^{\frac{4}{Q-1}}\left(v-v_{\mu}\right) \phi d z+\int_{B_{\mu}} v^{\frac{2}{Q-1}}\left(v-v_{\mu}\right) \phi d z^{\prime}\right]+I_{\varepsilon} .
\end{aligned}
$$

Moreover, if we set $C_{\mu}^{2}=C_{\mu}^{1} \cdot \sup \left\{v(x, y, t, \lambda)^{\frac{4}{Q-1}} \rho^{4}+v(x, y, t, \lambda)^{\frac{2}{Q-1}} \rho^{2} \mid t \geq \mu\right\}$, then we infer from the decay estimate of $v$ that $C_{\mu}^{2}$ is still well-defined for $\mu>0$ and is nonincreasing in $\mu$. So it follows from the above inequality that

$$
\begin{aligned}
& \int_{\Sigma_{\mu} \cap\left\{2 \varepsilon \leq\left\|z \circ p_{\mu}^{-1}\right\| \leq \frac{1}{\varepsilon}\right\}}\left|\nabla_{\hat{H}}\left(v-v_{\mu}\right)^{+}\right|^{2} d z \\
& \leq C_{\mu}^{2}\left[\int_{A_{\mu}} \frac{1}{\rho^{4}}\left(v-v_{\mu}\right) \phi d z+\int_{B_{\mu}} \frac{1}{\rho^{2}}\left(v-v_{\mu}\right) \phi d z^{\prime}\right]+I_{\varepsilon} \\
& \leq C_{\mu}^{2}\left[\int_{A_{\mu}} \frac{1}{\rho^{4}}\left[\left(v-v_{\mu}\right)^{+}\right]^{2} \eta_{\varepsilon}^{2} d z+\int_{B_{\mu}} \frac{1}{\rho^{2}}\left[\left(v-v_{\mu}\right)^{+}\right]^{2} \eta_{\varepsilon}^{2} d z^{\prime}\right]+I_{\varepsilon} \\
& \leq C_{\mu}^{2}\left(\int_{A_{\mu}} \frac{1}{\rho^{2 Q+2}}\right)^{\frac{2}{Q+1}} \cdot\left(\int_{\Sigma_{\mu}}\left[\left(v-v_{\mu}\right)^{+}\right]^{\frac{2 Q+2}{Q-1}} d z\right)^{\frac{Q-1}{Q+1}} \\
& +C_{\mu}^{2}\left(\int_{B_{\mu}} \frac{1}{\rho^{2 Q}}\right)^{\frac{1}{Q}}\left(\int_{\partial \Sigma_{\mu}}\left[\left(v-v_{\mu}\right)^{+}\right]^{\frac{2 Q}{Q-1}} d z^{\prime}\right)^{\frac{Q-1}{Q}}+I_{\varepsilon} .
\end{aligned}
$$


Next we claim that $I_{\varepsilon} \rightarrow 0$ as $\varepsilon \rightarrow 0$. In fact, if we denote $B_{\varepsilon}=\left\{z \in \Sigma_{\mu}: \varepsilon<\right.$ $\left\|z \circ p_{\mu}^{-1}\right\|<2 \varepsilon$ or $\left.\frac{1}{\varepsilon}<\left\|z \circ p_{\mu}^{-1}\right\|<\frac{2}{\varepsilon}\right\}$, then we get

$$
\int_{B_{\varepsilon}}\left|\nabla_{\hat{H}} \eta_{\varepsilon}\right|^{Q+1} d z \leq C
$$

Hence, we infer from Hölder inequality that

$I_{\varepsilon} \leq\left(\int_{B_{\varepsilon}}\left[\left(v-v_{\mu}\right)^{+}\right]^{2^{\#}} d z\right)^{\frac{2}{2 \#}} \cdot\left(\int_{B_{\varepsilon}}\left|\nabla_{\hat{H}} \eta_{\varepsilon}\right|^{Q+1} d z\right)^{\frac{2}{Q+1}} \leq C\left(\int_{B_{\varepsilon}}\left[\left(v-v_{\mu}\right)^{+}\right]^{2^{\#}} d z\right)^{\frac{2}{2 \#}} \rightarrow 0$ as $\varepsilon \rightarrow 0$.

Finally, let $\varepsilon \rightarrow 0$ in equation (3.7), and set $C_{\mu}=C_{\mu}^{2}\left(S+S_{T}\right)$ with $S$ and $S_{T}$ being the Sobolev constant and Sobolev trace inequality constant respectively, then we get

$$
\begin{aligned}
& \int_{\Sigma_{\mu}}\left|\nabla_{\hat{H}}\left(v-v_{\mu}\right)^{+}\right|^{2} d z \\
& \leq C_{\mu}^{2}\left(\int_{A_{\mu}} \frac{1}{\rho^{2 Q+2}}\right)^{\frac{2}{Q+1}} \cdot\left(\int_{\Sigma_{\mu}}\left[\left(v-v_{\mu}\right)^{+}\right]^{\frac{2 Q+2}{Q-1}} d z\right)^{\frac{Q-1}{Q+1}} \\
& +C_{\mu}^{2}\left(\int_{B_{\mu}} \frac{1}{\rho^{2 Q}}\right)^{\frac{1}{Q}}\left(\int_{\partial \Sigma_{\mu}}\left[\left(v-v_{\mu}\right)^{+}\right]^{\frac{2 Q}{Q-1}} d z^{\prime}\right)^{\frac{Q-1}{Q}} \\
& \leq C_{\mu}\left[\left(\int_{A_{\mu}} \frac{1}{\rho^{2 Q+2}}\right)^{\frac{2}{Q+1}}+\left(\int_{B_{\mu}} \frac{1}{\rho^{2 Q}}\right)^{\frac{1}{Q}}\right] \cdot\left(\int_{\Sigma_{\mu}}\left|\nabla_{\hat{H}}\left(v-v_{\mu}\right)^{+}\right|^{2} d z\right) .
\end{aligned}
$$

Moveover, it follows from the above procedure that $C_{\mu}$ satisfies all the requirements in the lemma.

Before we continue the proof of Theorem 1.1, we give some comment on this Lemma. As we notified in the introduction of this paper, since $f, g$ are only assumed to be continuous, the solution is usually not of $C^{2}$ class, hence the maximum principles in differential forms do not work. Thanks to the inequality of this Lemma, it can play the same role as the maximum principle. In fact, if we can prove

$$
C_{\mu}\left[\left(\int_{A_{\mu}} \frac{1}{\rho^{2 Q+2}}\right)^{\frac{2}{Q+1}}+\left(\int_{B_{\mu}} \frac{1}{\rho^{2 Q}}\right)^{\frac{1}{Q}}\right]<1
$$

then we get $v \leq v_{\mu}$, the same conclusion as the maximum principle in a differential form implies.

The next lemma shows that we can start the moving plane for $\mu$ sufficient large.

Lemma 3.2. Under assumptions of Theorem 1.1, there exists $\mu_{0}>0$, such that for all $\mu \geq \mu_{0}$, we have $v \leq v_{\mu}$ in $\Sigma_{\mu}$.

Proof. The conclusion of this lemma is a direct corollary of Lemma 3.1. In fact, by the decay behavior of $v$, see equation (3.1), we can choose $\mu_{0}$ large enough such that

$$
C_{\mu}\left[\left(\int_{A_{\mu}} \frac{1}{\rho^{2 Q+2}}\right)^{\frac{2}{Q+1}}+\left(\int_{B_{\mu}} \frac{1}{\rho^{2 Q}}\right)^{\frac{1}{Q}}\right]<\frac{1}{2}
$$

for all $\mu \geq \mu_{0}$, then equation (3.5) implies that

$$
\int_{\Sigma_{\mu}}\left|\nabla_{\hat{H}}\left(v-v_{\mu}\right)^{+}\right|^{2} d z=0 .
$$


The assertion follows.

Now we move the plane from the right to the left and suppose this process stops at $\mu_{1}$. More precisely, we define

$$
\mu_{1}=\inf \left\{\mu \mid v \leq v_{\bar{\mu}} \text { in } \Sigma_{\bar{\mu}} \text { for all } \bar{\mu} \geq \mu\right\},
$$

then we have the following

Lemma 3.3. If $\mu_{1}>0$, then $v(z) \equiv v_{\mu_{1}}(z)$ for all $z \in \Sigma_{\mu_{1}}$.

Proof. We prove the conclusion by contradiction. Suppose that $v \not \equiv v_{\mu_{1}}$, then we claim that the plane can be moved to the left a little. That is, we will show that there exists $\delta>0$, such that $v(z) \leq v_{\mu}(z)$ in $\Sigma_{\mu}$ for all $\mu \in\left[\mu_{1}-\delta, \mu_{1}\right]$. This contradicts the choice of $\mu_{1}$.

Now we prove our claim. Suppose that $v(z) \not \equiv v_{\mu_{1}}(z)$, then we infer from the continuity that $v(z) \leq v_{\mu_{1}}(z)$. Moreover, we have

$$
\begin{aligned}
h\left(\rho^{Q-1} v\right) v^{\frac{Q+3}{Q-1}} & =\frac{f\left(\rho^{Q-1} v\right)}{\rho^{Q+3}} \leq \frac{f\left(\rho^{Q-1} v_{\mu_{1}}\right)}{\rho^{Q+3}}=\frac{f\left(\rho^{Q-1} v_{\mu_{1}}\right)}{\left[\rho^{Q-1} v_{\mu_{1}}\right]^{\frac{Q+3}{Q-1}}} v_{\mu_{1}}^{\frac{Q+3}{Q-1}} \\
& \leq \frac{f\left(\rho_{\mu_{1}}^{Q-1} v_{\mu_{1}}\right)}{\left[\rho_{\mu_{1}}^{Q-1} v_{\mu_{1}}\right]^{Q+3}} v_{\mu_{1}}^{\frac{Q+3}{Q-1}}=h\left(\rho_{\mu_{1}}^{Q-1} v_{\mu_{1}}\right) v_{\mu_{1}}^{\frac{Q+3}{Q-1}},
\end{aligned}
$$

where the first inequality holds since $f$ is nondecreasing, the second inequality is a consequence of (ii) in Theorem 1.1.

This equation implies

$$
-L v \leq-L v_{\mu_{1}}
$$

then we infer from the maximum principle in [6] that $v<v_{\mu_{1}}$ in $\Sigma_{\mu_{1}}$ since $v \not \equiv$ $v_{\mu_{1}}$. Moreover, since $\frac{1}{\rho^{2 Q+2}} \chi_{A_{\mu}} \rightarrow 0, \frac{1}{\rho^{2 Q}} \chi_{B_{\mu}} \rightarrow 0$ a.e. as $\mu \rightarrow \mu_{1}$ and $\frac{1}{\rho^{2 Q+2}} \chi_{A_{\mu}} \leq$ $\frac{1}{\rho^{2 Q+2}} \chi_{\mu_{1}-\delta}, \frac{1}{\rho^{2 Q}} \chi_{B_{\mu}} \leq \frac{1}{\rho^{2 Q}} \chi_{\mu_{1}-\delta}$ for $\mu \in\left[\mu_{1}-\delta, \mu_{1}\right]$ and some $\delta>0$, then we infer from the dominated convergence theorem that

$$
\int_{A_{\mu}} \frac{1}{\rho^{2 Q+2}} d z \rightarrow 0
$$

and

$$
\int_{B_{\mu}} \frac{1}{\rho^{2 Q}} d z^{\prime} \rightarrow 0
$$

as $\mu \rightarrow \mu_{1}$. In particular, there exists $\delta>0$, such that

$$
C_{\mu}\left[\left(\int_{A_{\mu}} \frac{1}{\rho^{2 Q+2}}\right)^{\frac{2}{Q+1}}+\left(\int_{B_{\mu}} \frac{1}{\rho^{2 Q}}\right)^{\frac{1}{Q}}\right]<\frac{1}{2}
$$

for all $\mu \in\left[\mu_{1}-\delta, \mu_{1}\right]$. Then we infer from Lemma 3.1 that $v \leq v_{\mu}$ for all $\mu \in$ $\left[\mu_{1}-\delta, \mu_{1}\right]$. This contradicts the definition of $\mu_{1}$.

Proposition 3.4. Let $u, f, g$ as in Theorem 1.1 and suppose that $u$ is positive. Let $v$ be the $C R$ inversion of $u$ centered at a point $p=\left(0,0, t_{0}, 0\right)$, then $v$ is symmetric with respect to $T_{t_{0}}$.

Proof. To prove that $v$ is symmetric with respect to $T_{t_{0}}$, we use the method of moving plane as above. We first make a $\mathrm{CR}$ inversion at $p=\left(0,0, t_{0}, 0\right)$, then we carry out the procedure as the above and get the corresponding $\mu_{1}=\mu_{1}\left(t_{0}\right)$. If $\mu_{1}>t_{0}$, then it follows from Lemma 3.3 that $v$ is symmetric with respect to $T_{\mu_{1}}$. But the symmetry together with equation (3.2) and equation (3.3) imply that $\left\|z_{\mu}\right\|=\|z\|$ 
since either $h$ or $k$ is not a constant, which is impossible. Hence we get that $\mu_{1} \leq t_{0}$. Similarly, we can also perform the moving plane procedure from the left and find a corresponding $\mu_{1}^{\prime} \geq t_{0}$. Finally, we infer from $v_{\mu_{1}}(z) \geq v(z)$ and $v_{\mu_{1}^{\prime}}(z) \leq v(z)$ that $\mu_{1}=\mu_{1}^{\prime}=t_{0}$. That is, $v$ hence $u$ is symmetric with respect to $T_{t_{0}}$.

Proof of Theorem 1.1. By Proposition 3.4, we conclude that the CR inversion function $v$ of $u$ at $p=\left(0,0, t_{0}, 0\right)$ is symmetric with respect to the $T_{t_{0}}$ for any $t_{0} \in \mathbf{R}$. Since $t_{0}$ is arbitrary, then we conclude that $u$ is independent of $t$. However, this implies that $u$ satisfies the equation

$$
\left\{\begin{array}{l}
-\Delta u=f(u) \quad \text { in } \mathbf{R}_{+}^{2 n+1} \\
-\frac{\partial u}{\partial \lambda}=g(u) \quad \text { on } \partial \mathbf{R}_{+}^{2 n+1}
\end{array}\right.
$$

Since $f, g$ is nondecreasing in $(0, \infty)$ and

$$
\frac{f(t)}{t^{\frac{2 n+3}{2 n-1}}}=\frac{f(t)}{t^{\frac{Q+2}{Q-2}}} t^{\frac{Q+3}{Q-1}-\frac{2 n+3}{2 n-1}}, \quad \frac{g(t)}{t^{\frac{2 n+1}{2 n-1}}}=\frac{g(t)}{t^{\frac{Q+1}{Q-1}}} t^{\frac{Q+1}{Q-1}-\frac{2 n+1}{2 n-1}}
$$

is decreasing in $t$, then Theorem 1.1 in [20] implies that $u \equiv c$ with $f(c)=g(c)=$ 0 .

Acknowledgement. The author would like to thank the anonymous referee for his/her useful suggestions, which indeed improved this paper.

\section{References}

[1] Birindelli, I., I. C. Dolcetta, and A. Cutri: Liouville theorems for semilinear equations on the Heisenberg group. - Ann. Inst. H. Poincaré Anal. Non Linéaire 14, 1997, 295-308.

[2] Birindelli, I., and E. LANCONELli: A negative answer to a one-dimensional symmetry problem in the Heisenberg group. - Calc. Var. Partial Differential Equations 18, 2003, 357-372.

[3] Birindelli, I., and J. Prajapat: Nonlinear Liouville theorems in the Heisenberg group via the moving plane method. - Comm. Partial Differential Equations 24, 1999, 1875-1890.

[4] Birindelli, I., and J. Prajapat: Monotonicity results for nilpotent stratified groups. - Pacific J. Math. 204, 2002, 1-17.

[5] Chen, W., and C. Li: Classification of solutions of some nonlinear elliptic equations. - Duke Math. J. 63, 1991, 615-622.

[6] Cinti, E., and J. TAN: A nonlinear Liouville theorem for fractional equations in the Heisenberg group. - J. Math. Anal. Appl. 433, 2016, 434-454.

[7] Damascelli, L., and F. Gladiali: Some nonexistence results for positive solutions of elliptic equations in unbounded domains. - Rev. Mat. Iberoam. 20, 2004, 67-86.

[8] Frank, R., M. González, D. Monticelli, and J. Tan: An extension problem for the CR fractional Laplacian. - Adv. Math. 270, 2015, 97-137.

[9] Garofalo, N., and D. Vassilev: Symmetry properties of positive entire solutions of Yamabetype equations on groups of Heisenberg type. - Duke Math. J. 106, 2001, 411-448.

[10] Gidas, B., and J. SpRUck: Global and local behavior of positive solutions of nonlinear elliptic equations. - Comm. Pure Appl. Math. 24, 1981, 525-598.

[11] Liu, J., Y. Guo, and Y. Zhang: Liouville-type theorems for polyharmonic systems in $\mathbf{R}^{N}$. J. Differential Equations 225, 2006, 685-709.

[12] Guo, Y., and J. Liu: Liouville type theorems for positive solutions of elliptic system in $\mathbf{R}^{N}$. - Comm. Partial Differential Equations 33, 2008, 263-284.

[13] Guo, Y., and J. Liv: Liouville-type theorems for polyharmonic equations in $\mathbf{R}^{N}$ and in $\mathbf{R}_{+}^{N}$. - Proc. Roy. Soc. Edinburgh Sect. A 138, 2008, 339-359. 
[14] Hörmander, L.: Hypoelliptic second order differential equations. - Acta Math. 119, 1967, $147-171$.

[15] Terracini, S.: Symmetry properties of positives solutions to some elliptic equations with nonlinear boundary conditions. - Differential Integral Equations 8, 1995, 1911-1922.

[16] Terracini, S.: On positive entire solutions to a class of equations with a singular coefficient and critical exponent. - Adv. Differential Equations 1, 1996, 241-264.

[17] XU, L.: Semi-linear Liouville theorems in the Heisenberg group via vector field methods. - J. Differential Equations 247, 2009, 2799-2820.

[18] YU, X.: Liouville type theorems for integral equations and integral systems. - Calc. Var. Partial Differential Equations 46, 2013, 75-95.

[19] YU, X.: Liouville type theorem in the Heisenberg group with general nonlinearity. - J. Differential Equations 254, 2013, 2173-2182.

[20] YU, X.: Liouville type theorem for nonlinear elliptic equation with general nonlinearity. Discrete Contin. Dyn. Syst. 34, 2014, 4947-4966.

Received 5 May 2016• Received 8 August 2016 • Accepted 23 December 2016 